\title{
Marine bioadhesives: opportunities and challenges
}

\author{
Kamla Pathak*,1 (iD \\ ${ }^{1}$ Faculty of Pharmacy, Uttar Pradesh University of Medical Sciences, Saifai, Etawah 2016130, Uttar Pradesh, India \\ *Author for correspondence: kamlapathak5@gmail.com
"Mussel adhesive proteins, polysaccharides and exopolysaccharides are distinct categories of bioadhesives obtained from sea."

First draft submitted: 12 September 2019; Accepted for publication: 2 October 2019; Published online: 24 October 2019 Keywords: alginates $\bullet$ chitosan $\bullet$ chitosan derivatives $\bullet$ composite bioadhesives $\bullet$ exopolysaccharides $\bullet$ marine bioad-
hesives $\bullet$ polysaccharides $\bullet$ proteins

Marine invertebrates produce adhesives that retain adhesion even in harsh conditions of sea water. Mussel adhesive proteins, polysaccharides and exopolysaccharides are distinct categories of bioadhesives obtained from sea. Of these, the former two have been extensively explored for developing a variety of drug-delivery systems, including nanoformulations. Combination of adhesives and development of composite bioadhesives are newer approaches to overcome issues related to use of solitary adhesive in formulations.

The ability of marine invertebrates to produce adhesives that retain adhesion, in the presence of harsh sea water conditions, has garnered considerable attention of pharmaceutical researchers. Mussel adhesive proteins, polysaccharides and exopolysaccharides are distinct groups of bioadhesives obtained from the sea [1]. Many therapeutic agents can be delivered via the drug-delivery systems containing these bioadhesives, including anti-inflammatory agents, antihypertensive agents, peptides and low molecular weight proteins, etc. The bioadhesives can be utilized for local drug delivery (topical) and requirements for successful bioadhesive devices for sustained topical delivery of active ingredients include: establishing and maintaining intimate contact with the site of application; ensuring sustained/controlled delivery of the drug in pathological skin conditions; be sufficiently adhesive and cohesive; be nontoxic and nonirritating; and allowing easy termination of therapy [2].

\section{Marine polysaccharides}

Research efforts on algal bioadhesives have led to the isolation of polysaccharides that are promising biomaterials, especially as adhesives in ophthalmic therapies. For the purpose of drug delivery, many polysaccharides, including chitin, chitosan, agar, alginate and carrageenans, have been investigated. Chitosan, a biopolymer derived from chitin, has wide pharmaceutical applications, ranging from capability to form gels, films, micro/nanoparticles and beads, as wound dressing and as a carrier for drug/gene delivery [3]. It has been utilized for designing mucoadhesive dosage forms but suffers from its limited mucoadhesive strength and limited water solubility at neutral and basic $\mathrm{pH}$. These issues have been tackled by designing chitosan derivatives such as carboxymethyl chitosan, chitosanEDTA, glycol chitosan, chitosan-catechol and cyclodextrin-chitosan [4]. The derivatization of chitosan to improve its mucoadhesive properties has been explained in several publications. Few of them have demonstrated potential in transmucosal delivery, for example ChiSys ${ }^{\circledR}$ as a platform for nasal vaccination [5] and Lacimera ${ }^{\circledR}$ eye drops [6].

Alginates are linear polysaccharides obtained from seaweeds and marine algae, utilized in drug delivery, tissue engineering applications and immobilization of cells and enzymes owing to their ability to crosslink through bivalent cations. Alginates instantaneously form gel-spheres with divalent ions at $\mathrm{pH}>6$ and are widely utilized for the microencapsulation of drugs. However, high viscosity gels are formed when the alginates are subjected to low $\mathrm{pH}$ values [7]. The ability of alginates to form $\mathrm{pH}$-triggered gels has been widely used to develop ophthalmic drugdelivery systems. Furthermore, the ability of alginates to form microcapsules on cross-linking has been specifically explored for the oral delivery of low molecular weight proteins [8]. 
Alginates can be combined with other bioadhesives to develop novel efficacious drug-delivery systems and the concept has garnered considerable interest among pharmaceutical formulation researchers. For example, the chitosan-alginate microbeads for sustained vaginal delivery of chlorhexidine digluconate, jackfruit seed starchalginate microspheres of metformin $\mathrm{HCl}$ [9], tamarind seed polysachharide-alginate beads for gliclazide oral delivery, etc. [10]. Alginates have also been utilized for drug-delivery systems based on composite technology. For example, montmorillonite-alginate nanocomposites have been developed for sustained delivery of vitamin B1 and B6 [11]. Modulated drug release was achievable by incorporating $\mathrm{pH}$-independent hydrocolloids gelling agents or by addition of chitosan [12].

Large number of mucoadhesive systems based on alginates have been developed, such as a mucoadhesive vaginal tablet of acriflavine for veterinary purposes. The main drawbacks of alginate formulations are their rapid erosion at neutral $\mathrm{pH}$ and reduction in mucoadhesion on cross-linking with divalent cations. In order to strengthen the mucoadhesive property of alginates, these have been chemically derivatized; for example, alginate-polyethyleneglycol acrylate has gelation ability of alginate combined with mucoadhesive strength of acrylate functionality of polyethylene glycol [13]. Laurienzo et al. attempted modification of sodium alginate with amines and/or acid moieties that resulted in optimization of drug delivery properties, namely, the drug release rate, modulation of polysaccharide erosion pattern and adhesion to the biological substrates [14]. Bernkop et al. demonstrated an improvement of mucoadhesive properties and the release retardant properties of sodium alginate due to covalent linkage of cysteine [15]. The sustainment of drug release and improved mucoadhesion is exemplified by matrix tablets based on sodium alginate-cysteine conjugate containing tramadol hydrochloride as a model drug [16]. Furthermore, the low toxicity of these new excipients provides immense scope for utilization of thiolated polymers in mucoadhesive drug-delivery systems.

\section{Adhesive proteins}

Mussel adhesive proteins are recognized as powerful adhesives, which are stronger compared with the polymerbased adhesives. Most importantly, mussel adhesive proteins retain adhesive capacity in wet environments, which is not demonstrated by polymer-based adhesives. To date no polymer-based adhesives have been produced that can retain adhesion in an underwater environment. The strong adhesion of marine mussels under the sea inspired the development of several water-resistant adhesives. The catechol groups are present in large quantities in mussel adhesive proteins, which contribute to the outstanding adhesion of mussels on many different surfaces [17]. Catechols interact with biological surfaces, including mucus. These findings inspired researchers to examine catechol-chitosan mucoadhesive systems for drug delivery via oral, buccal and rectal routes. Catechol-modified chitosan hydrogel crosslinked by genipin is a promising mucoadhesive and biocompatible hydrogel system for buccal drug delivery [18]. Chitosan-catechol mucoadhesive gel allowed sulfasalazine delivery by rectal route more effectively and safely compared with oral administration [19].

It may become imperative for the researchers to amalgamate several adhesive proteins to develop improvised bioadhesive materials for novel applications including drug, protein and gene delivery. Novel drug delivery formulations consisting of polyphenolic proteins from mussels and marine origin polysaccharides are promising, especially as adhesive in ophthalmic therapies.

\section{Marine exopolysacchrides}

Exopolysaccharides are obtained from marine bacteria. However, most of these are poorly understood and only a few have been fully characterized. Of the various pharmaceutical roles of microbial exopolysaccharides, assistance in attachment to sea surfaces is of particular interest. Exopolymers of marine Vibrio MH3 are involved in reversible attachment. The cross-linking of adjacent polysaccharide chains aids in permanent adhesion [20]. Exopolysaccharides are yet to be explored for drug delivery. Interest is being directed toward extreme marine environments, which could represent a large source of unidentified bacteria.

Dedicated research by marine scientists has afforded discovery and production of many marine bioadhesives of pharmaceutical interest. The possibility of chemical modification, addition of biodegradable additives and production of hybrid bioadhesives permits modulation of properties of bioadhesives and new possibilities, particularly in the pharmaceutical area. 
Financial \& competing interests disclosure

The author has no relevant affiliations or financial involvement with any organization or entity with a financial interest in or financial conflict with the subject matter or materials discussed in the manuscript. This includes employment, consultancies, honoraria, stock ownership or options, expert testimony, grants or patents received or pending, or royalties.

No writing assistance was utilized in the production of this manuscript.

References

1. Waite JH. Nature's underwater adhesive specialist. Int. J. Adhes. Adhes. 7, 9-14 (1987)

2. Paola L. Marine polysaccharides in pharmaceutical applications: an overview. Mar. Drugs 8, 2435-2465 (2010).

3. Ali A, Ahmed S. A review on chitosan and its nanocomposites in drug delivery. Int. J. Biol. Macromol. 109, 273-286 (2018).

4. Ways TMM, Lau WM, Khutoryanskiy VV. Chitosan and its derivatives for application in mucoadhesive drug-delivery systems. Polymers 10, 267 (2018).

5. Watts P, Smith A, Hinchcliffe M. ChiSys ${ }^{\circledR}$ as a chitosan-based delivery platform for nasal vaccination. Mucos. Deliv. Biopharmaceut. 499-516 (2014).

6. Bonengel S, Bernkop-Schnürch A. Thiomers - from bench to market. J. Control. Rel. 195, 120-129 (2014).

7. Patil SB, Sawant KK. Development, optimization and in vitro evaluation of alginate mucoadhesive microspheres of carvedilol for nasal delivery. J. Microencapsul. 26(5), 432-443 (2009).

8. Hua S, Ma H, Li X et al. pH-sensitive sodium alginate/poly(vinyl alcohol) hydrogel beads prepared by combined $\mathrm{Ca}^{2+}$ crosslinking and freeze-thawing cycles for controlled release of diclofenac sodium. Int. J. Biol. Macromol. 46, 517-523 (2010).

9. Chatterjee B, Amalina N, Sengupta P, Mandal UK. Mucoadhesive polymers and their mode of action: recent update. J. Appl. Pharm. Sci. 7(5), 195-203 (2017)

10. Nayak AK, Pal D. Formulation optimization and evaluation of jackfruit seed starch-alginate mucoadhesive beads of metformin $\mathrm{HCl}$. Int J. Biol. Macromol. 49, 264-272 (2013).

11. Pal D, Nayak AK. Novel tamarind seed polysaccharide-alginate mucoadhesive microspheres for oral gliclazide delivery: in vitro-in vivo evaluation. Drug Deliv. 19, 123-131 (2012).

12. Kevadiya BD, Joshi GV, Patel HA, Ingole PG, Mody HM, Bajaj HC. Montmorillonite-alginate nanocomposites as a drug-delivery system: intercalation and in vitro release of vitamin B1 and vitamin B6. J. Biomater. Appl. 25, 161-177 (2010).

13. Tapia $\mathrm{C}$, Escobar Z, Costa $\mathrm{E}$ et al. Comparative studies on polyelectrolyte complexes and mixtures of chitosan-alginate and chitosan-carrageenan as prolonged diltiazem chlorhydrate release systems. Eur. J. Pharm. Biopharm. 57, 65-75 (2004).

14. Davidovich-Pinhas M, Bianco-Peled H. Alginate-PEGAc: a new mucoadhesive polymer. Acta Biomater. 7, 625-633 (2011).

15. Laurienzo $\mathrm{P}$, Malinconico M, Mattia G et al. Novel alginate-acrylic polymers as a platform for drug delivery. J Biomed. Mater. Re. A. 78, 523-531 (2006).

16. Bernkop-Schnürch A, Clausen AE, Hnatyszyn M. Thiolated polymers: synthesis and in vitro evaluation of polymer-cysteamine conjugates. Int. J. Pharm. 226, 185-194 (2001).

17. Jindal $\mathrm{AB}$, Wasnik MN, Nair HA. Synthesis of thiolated alginate and evaluation of mucoadhesiveness, cytotoxicity and release retardant properties. Indian J. Pharm. Sci. 72(6), 766-774 (2010).

18. Dove J, Sheridan P. Adhesive protein from mussels: possibilities for dentistry, medicine, and industry. J. Am. Dent. Assoc. 112, 879-883 (1986).

19. Xu J, Strandman S, Zhu JX, Barralet J, Cerruti M. Genipin-crosslinked catechol-chitosan mucoadhesive hydrogels for buccal drug delivery. Biomaterials 37, 395-404 (2015).

20. Xu J, Tam M, Samaei $S$ et al. Mucoadhesive chitosan hydrogels as rectal drug delivery vessels to treat ulcerative colitis. Acta Biomater. 48, 247-257 (2017). 


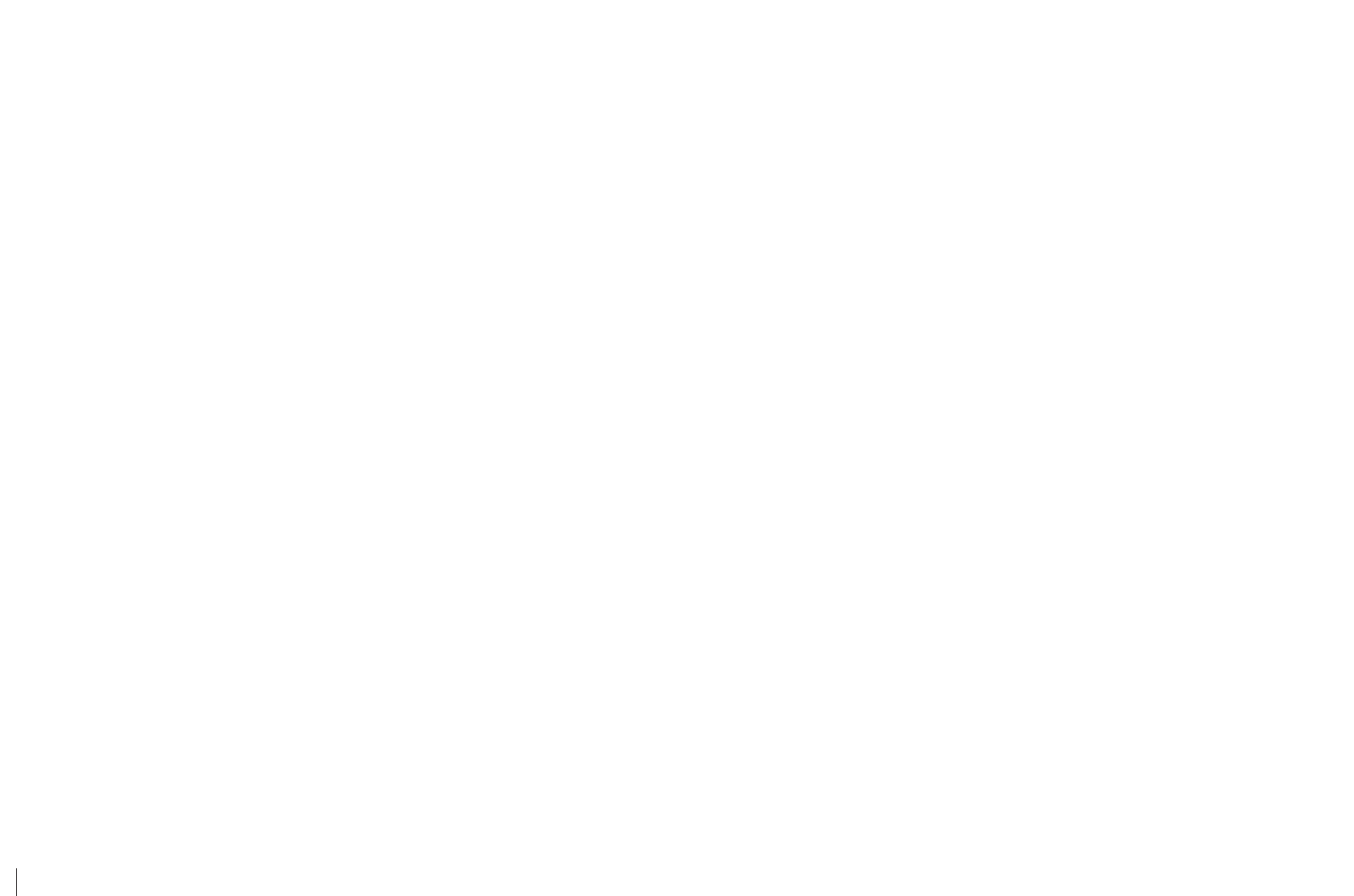

